\title{
Motor response vigour and fixations reflect subjective preferences during intertemporal choice
}

\author{
Elke Smith, Jan Peters \\ Department of Psychology, Biological Psychology, University of Cologne, Germany \\ e.smith@uni-koeln.de jan.peters@uni-koeln.de
}

\begin{abstract}
Value-based decision-making is of central interest in cognitive neuroscience and psychology, as well as in the context of neuropsychiatric disorders characterised by decision-making impairments. Studies examining (neuro-)computational mechanisms underlying choice behaviour typically focus on participants' decisions. However, there is increasing evidence that option valuation might also be reflected in motor response vigour and eye movements, implicit measures of subjective utility.

To examine motor response vigour and visual fixation correlates of option valuation in intertemporal choice, we set up a task where the participants selected an option by pressing a grip force transducer, simultaneously tracking fixation shifts between options. As outlined in our preregistration (https:// osf.io/k6jct), we used hierarchical Bayesian parameter estimation to model the choices assuming hyperbolic discounting, compared variants of the softmax and drift diffusion model, and assessed the relationship between response vigour and the estimated model parameters.

The behavioural data were best explained by a drift diffusion model specifying a non-linear scaling of the drift rate by the subjective value differences. Replicating previous findings (Green et al., 1997; Wagner et al., 2020), we found a magnitude effect for temporal discounting, such that higher rewards were discounted less. This magnitude effect was further reflected in response vigour, such that stronger forces were exerted in the high vs. the low magnitude condition. Bayesian hierarchical linear regression further revealed higher grip forces, faster response times and a lower number of fixation shifts for trials with higher subjective value differences.

Our data suggest that subjective utility or implicit valuation is reflected in response vigour during intertemporal choice. Taking into account response vigour might thus provide deeper insight into decision-making, reward valuation and maladaptive changes in these processes, e.g. in the context of neuropsychiatric disorders.
\end{abstract}

Keywords - Response vigour, grip force, intertemporal choice, value-based decision-making 


\section{INTRODUCTION}

Motivation entails the willingness to perform effortful actions in order to obtain rewards. Individuals normally adapt the level of effort expended to the expected utility of a reward. An adequate adjustment of effort to expected utility is crucial to ensure reward receipt, whilst avoiding unnecessary energy expenditure. Whether a reward is worth a given effort depends on its expected (subjective) utilty. The expected utility of a reward does not equal its utility in an absolute sense, but is contingent upon both intraindividual and external factors. For instance, rewards that are temporally more distant are typically devaluated, resulting in a preference for smaller, but sooner rewards, over larger, but later rewards, a process known as temporal discounting (Odum, 2011; Peters \& Büchel, 2009). Likewise, rewards associated with costs of physical effort are also devaluated, a process referred to as effort discounting (Klein-Flügge, Kennerley, Saraiva, Penny, \& Bestmann, 2015; Prévost, Pessiglione, Météreau, Cléry-Melin, \& Dreher, 2010).

The degree of discounting delayed rewards has been linked to a range of harmful behaviours and psychiatric conditions, including impulsivity, substance abuse and addiction (for a review, see Moreira \& Barbosa, 2019). For instance, individuals suffering from substance use disorders appear to be biased towards choosing immediate compared to delayed, but larger, rewards (Karakula et al., 2016; Yi, Mitchell, \& Bickel, 2010).

Key brain circuits involved in value-based decision-making include the medial prefrontal cortex and striatum. Here, brain activity correlates with subjective value in a variety of tasks, such as valuation of goods and intertemporal choice (Chib, Rangel, Shimojo, \& O'Doherty, 2009; Kable \& Glimcher, 2007; Levy \& Glimcher, 2011). The devaluation of rewards by both cognitive and physical effort appears to be associated with BOLD activation in mostly overlapping neural structures (Chong et al., 2017).

It is well established that midbrain dopaminergic neurons play a central role in decisionmaking and reward processing (Rogers, 2011; Schultz, 2010). Direct evidence for the involvement of dopamine in effort-based decision-making comes from studies in patients with Parkinson's disease (PD) and from pharmacological studies manipulating dopamine transmission. In patients with PD, effort-based decision-making appears to be disrupted - they have been found to exert less force for rewards compared to healthy controls, and to exert less force when being off compared to on their dopaminergic medication (Chong et al., 2015; Le Bouc et al., 2016). In turn, pharmacological enhancement of dopamine transmission via levodopa in healthy participants increased the force levels exerted to obtain high vs. low rewards. Following debriefing, none of the participants reported to excert higher forces to obtain high rewards (Michely et al., 2020), suggesting that the behaviour reflects an implicit motivational process (Michely et al., 2020).

Pessiglione and colleagues (2007) found that participants exert more force to obtain higher rewards even in cases where the rewards have only been presented subliminally. Also, across different social contexts (collaborative and competitive), force production was strongly related to subjective utility, and increased with absolute monetary value (Le Bouc \& Pessiglione, 2013). Further, subjective utility in value-based decision-making is reflected in eye movement vigour (Shadmehr, Reppert, Summerside, Yoon, \& Ahmed, 2019). For instance, as participants approach 
their decision, eye movement vigour (i.e. peak velocity of a saccade as a function of amplitude) becomes greater for the preferred reward option, and the difference in eye movement vigour is closely linked to the difference in assigned subjective values of the options (Reppert, Lempert, Glimcher, \& Shadmehr, 2015).

While value-based decision-making is a complex process requiring information integration, value computation and comparison, in most experimental settings, the process of evaluating a reward's utility is often inferred from the participants' choices only. However, from the above findings it appears that measures of response vigour may provide additional insights into motivation and value-based decision-making rather than measures of choice behaviour alone. In the present study, we therefore investigated if measures of response vigour, specifically gaze shifting and handgrip force applied during choice selection, may serve as implicit measure of outcome utility and decision conflict during intertemporal choice. In contrast to the incentive force task used by Pessiglione and colleagues $(2007$; 2013), where the force applied was directly related to the payout and visually fed back to the participants, we captured implicit motivational processes by keeping the amount of force produced hidden from the participants and unrelated to the payout. Besides being instrumental in obtaining a reward, the allocation of effort may also be a correlate of the underlying evaluation process. We further included an experimental manipulation known to substantially affect reward valuation during temporal discounting, the magnitude effect (Ballard et al., 2017; Green et al., 1997). This effect describes the reduction in discount rates (i.e. the increase in subjective utility) that occurs during intertemporal choice for increasing reward amounts, and we explored whether this effect is also reflected in the handgrip response.

Models of value-based decision-making, including temporal discounting, typically implement action selection using the softmax function (Sutton \& Barto, 2018). We extend this approach by jointly modelling the choices and response times (RTs) with the drift diffusion model (Ratcliff \& McKoon, 2008, DDM), a form of sequential sampling model for two-alternative forced choice tasks. The drift diffusion model assumes that choices are driven by a noisy accumulation process, which terminates as soon as the level of accumulated evidence has reached one of two response boundaries. The model's strength lies in the incorporation of both choices and RTs in the model estimation. It has proven to be a useful model in explaining choice behaviour and RTs during value-based decision-making in our and other's prior work (Fontanesi, Gluth, Spektor, \& Rieskamp, 2019; Krajbich, Armel, \& Rangel, 2010; Peters \& D’Esposito, 2020; Shahar et al., 2019; Wagner et al., 2020).

We analysed the relationship between the subjective value differences as derived from the estimated drift diffusion model parameters and the force applied and fixation shifts during response selection. Further, we assessed the relationship between decision conflict and response vigour. As outlined in the preregistration of our study (https://osf.io/k6jct), we tested the following hypotheses:

(i) Delay influences reward evaluation: Participants show a preference for smaller, but sooner rewards over larger, but later rewards (temporal discounting). 
(ii) Subjective utility modulates response times and grip force: Faster response times and stronger effort (handgrip force) for choices with high utility (subjective value).

(iii) Decision conflict is reflected in the measures of response vigour: Longer deliberation (response time), less vigour (grip force) and more frequent fixation shifts between the options during high-conflict decisions (choice options with similar subjective value).

(iv) Higher rewards are discounted less and elicit more effort: Lower discount rates, faster response times, and greater vigour (grip force) for larger rewards (magnitude effect).

\section{Methods}

\subsection{Sample}

Based on the effect sizes of previous studies reporting a magnitude effect for temporal discounting and handgrip force, respectively (Ballard et al., 2017; Green et al., 1997; Pessiglione et al., 2007), a power analysis yielded a sample size of $\mathrm{N}=20$ (effect size Cohen's $d_{\mathrm{z}}=1.1698$ and $d_{\mathrm{z}}=-0.7481$, respectively, $\alpha$ error probability $=.05$, power $=.95$, one-tailed paired $t$-test). We doubled the sample size and tested 42 participants in total. As two participants had to be excluded due to technical issues, the final sample consisted of $N=40$ participants (30 women, 34 right-handed, 39 with German Abitur, 1 with German Mittlere Reife or GCSE), aged 18 to $39(M=23.95, S D=4.90)$.

The participants were recruited through university bulletins, mailing lists and by word-ofmouth recommendation. Eligibility criteria included normal or corrected-to-normal vision and German as first language (or profound German language skills). Participants with strongly impaired vision, strabismus and psychiatric disorders were excluded. The study was approved by the local institutional review board and all participants provided informed written consent.

\subsection{Task}

The study was implemented as one-group, repeated-measures within-subject design, including two conditions. The participants performed 192 trials of an intertemporal choice task, whereby they had to choose between smaller-but-sooner (SS) and larger-but-later (LL) rewards. On one half of the trials, the SS reward was lower (10€, low condition), and on the other half the SS reward was higher ( $20 €$, high condition). The SS reward was always available immediately, while the LL reward consisted of combinations of sixteen percentages of the SS reward value [1.03 1.05 1.101 .151 .201 .251 .351 .451 .501 .701 .902 .202 .502 .903 .303 .80 ] and six delay periods [ 1713 3158 12]. The order of the trials and the assignment of the options to the left and right side of the screen were presented in randomised order. An option was preselected through visual fixation. For this purpose, we used an eyetracking system (SensoMotoric Instruments, Model: RED 500, sampling rate: $500 \mathrm{~Hz}$ ) to track the gaze patterns and to give real-time feedback to the participant, highlighting the currently fixated reward option. For highlighting the fixated option, the corresponding screen areas were defined as follows: Left area $<=\frac{1}{10}$ screen pixels of $\mathrm{x}$-coordinate $\mathrm{x} 4$, middle area $>=\frac{1}{10}$ screen pixels of $\mathrm{x}$-coordinate $\mathrm{x} 4$ and $<=\frac{1}{10}$ screen pixels of 
$x$-coordinate $x 6$, right area $>=\frac{1}{10}$ screen pixels of $x$-coordinate $x 6$. The responses were logged using a hand dynamometer measuring grip force (BIOPAC Systems, Inc., Model: TSD121C, isometric range: 0 - $100 \mathrm{kgf}$ ). The measured variables included the participants' choices, response times, fixation shift patterns and handgrip force applied during response selection, as well as their maximum handgrip force.

\subsection{Data collection}

The measurements took place at the Psychology Department of the University of Cologne. The participants were financially reimbursed for participation and additionally received the payout from one randomly selected trial (restricted to maximum $40 €$ ). During testing, the participants were seated in a dimly lit, electrically and acoustically shielded room, with their head placed in a chinrest. Prior to the experiment, they were instructed to press the handgrip with maximal force three times in succession with their dominant hand. The procedure was disguised as calibration procedure. The participants were further instructed that, as long as the threshold for logging a response is reached, the level of force exerted is irrelevant to the task structure.

\subsection{Data analyses}

\subsubsection{Preprocessing}

All logfiles were checked for stereotypic response patterns (exclusively SS or LL choices), none were found. We excluded trials with response times below $200 \mathrm{~ms}$ or above $10 \mathrm{~s}$, and trials with maximum grip force values falling below the threshold for logging a response (technical issue with faulty signal on parallel port). In total, 139 trials (1.81\% of trials) from 26 participants were excluded. The gripforce data were further baseline-corrected to zero, normalised to each participant's maximal voluntary contraction (MVC, greatest force exerted over three contractions), and smoothed with a moving average of 50 samples.

\subsubsection{Computational modelling of behaviour}

Temporal discounting model

Ensuing from previous research on the effects of immediacy vs. delay on choice behaviour, we assume temporal discounting to be hyperbolic (Green, Myerson, \& Macaux, 2005; Mazur, 1987). We quantified the discount rates using a model-based approach of hyperbolic discounting. To capture the choice behaviour in both conditions within a single model, we fitted a single subjectspecific discount rate parameter $k$ (estimated in logarithmic space), modelling the discount rate in the low condition, plus a subject-specific parameter $s$, modelling the change in the discount rate from the low compared to the high condition.

$$
S V(L L)_{t}=\frac{A_{t}}{1+e^{k+I_{t} * s_{k}} * I R I_{t}}
$$


Here, $S V$ is the subjective (discounted) value of the delayed reward and $A$ is the amount of the LL reward on trial $t$. $K$ is the (subject-specific) discount rate for the low condition (in logarithmic space), $s$ is a (subject-specific) shift in $\log (k)$ from the low to high condition, $I$ is a condition indicator variable (zero for low trials, one for high trials), and IRI is the inter-reward-interval.

\section{Softmax choice rule}

The softmax choice rule models the probability of choosing the LL reward on trial $t$ as

$$
P(L L)_{t}=\frac{e^{\beta * S V\left(L L_{t}\right)}}{e^{\beta * S V\left(L L_{t}\right)}+e^{\beta * S V\left(S S_{t}\right)}}
$$

$S V$ is the subjective value of the LL option, and $\beta$ is an inverse temperature parameter, describing the stochasticity of the choices (for $\beta=0$ the choices are random, while as $\beta$ increases, the choices become increasingly dependent on the values of the options).

\section{Drift diffusion model}

We further modelled the participants' choices using the drift diffusion model (DDM), whereby the softmax choice rule is replaced by the drift diffusion choice rule. For the boundary definitions of the DDM, we applied stimulus coding, with the lower boundary defined as choosing the SS reward, and the upper boundary defined as choosing the LL reward. For this purpose, choices towards the lower boundary were multiplied by -1 . When using absolute RT cut-offs, single fast trials force model parameters to adapt these trials und hence lead to a poor model fit at the single-subject level (Peters \& D'Esposito, 2020). We therefore excluded each participant's slowest and fastest $2.5 \%$ trials from the analysis. The response time on trial $t$ is distributed following the Wiener first passage time (WFPT):

$$
R T_{t} \sim w f p t(\alpha, \tau, z, v)
$$

The parameter $\alpha$ reflects the boundary separation (modelling a speed-accuracy trade-off), $\tau$ is the non-decision time (modelling processing time unrelated to the decision process), $v$ is the drift rate (modelling the rate of evidence accumulation), and $z$ is the starting-point bias (modelling a bias towards one of the boundaries). Using the JAGS Wiener module (Wabersich \& Vandekerckhove, 2014), $z$ may range between 0 and 1 , whereby $z=.5$ indicates no bias in either direction, $z<.05$ indicates a bias towards the lower boundary (SS option), and $z>.05$ indicates a bias towards the upper boundary (LL option).

First, we fitted a null model $\left(\mathrm{DDM}_{0}\right)$ without value modulation. This model comprises four parameters $(\alpha, \tau, z$, and $v)$, which are constant across trials for each participant. To connect the drift diffusion model with the valuation model (see equation 1), we implemented two further models comprising a function which links the trial-by-trial variability in the drift rate $v$ to the 
value differences. First, we realised a linear model $\left(\mathrm{DDM}_{\text {lin }}\right)$, following Pedersen, Frank, and Biele (2017):

$$
v_{t}=v_{c o e f f} *\left(S V\left(L L_{t}\right)-S V\left(S S_{t}\right)\right)
$$

The parameter $v_{\text {coeff }}$ maps the value differences onto the drift rate $v$ and transforms these differences to the proper scale of the DDM (Pedersen et al., 2017). As a last step, we implemented a sigmoid model $\left(\mathrm{DDM}_{\mathrm{sig}}\right)$, entailing a non-linear transformation of the scaled value differences with an S-shaped function as proposed by Fontanesi, Gluth, Spektor, and Rieskamp (2019), where $S$ is a sigmoid function centred at zero with slope $m$ and asymptote $\pm v_{\max }$ :

$$
\begin{gathered}
v_{t}=S\left(v_{\text {coeff }} *\left(S V\left(L L_{t}\right)-S V\left(S S_{t}\right)\right)\right) \\
S(m)=\frac{2 * v_{\max }}{1+e^{-m}}-v_{\max }
\end{gathered}
$$

Ensuing from this model, we also realised a shift model $\left(\mathrm{DDM}_{\text {shift }}\right)$, including the parameters $s_{\alpha}$, $s_{\tau}, s_{z}, s_{v}, s_{v_{c o e f f}}$, and $s_{v_{\max }}$ to model changes in the parameter distributions from the low to high condition:

$$
\begin{gathered}
R T_{t} \sim w f p t\left(\alpha+I_{t} * s_{\alpha}, \tau+I_{t} * s_{\tau}, z+I_{t} * s_{z}, v+I_{t} * s_{v}\right) \\
v_{t}=S\left(v_{c o e f f}+I_{t} * s_{v_{\text {coeff }}} *\left(S V\left(L L_{t}\right)-S V\left(S S_{t}\right)\right)\right) \\
S(m)=\frac{2 *\left(v_{\max }+I_{t} * s_{v_{\max }}\right)}{1+e^{-m}}-\left(v_{\max }+I_{t} * s_{v_{\max }}\right)
\end{gathered}
$$

Since the drift rate depends on the absolute magnitudes of the values, which, in turn differ between the low and high condition, condition effects are somewhat difficult to interpret. Extending the modelling as set out in the preregistration plan, we therefore further compared the drift diffusion models using absolute vs. normalised values (normalised by the maximum value of the LL reward per magnitude condition).

\section{Decision conflict}

To assess the hypothesised relationship between decision conflict and response vigour, we considered two different operationalisations of decision conflict, based on 
(i) the softmax choice rule and choice probability of the chosen reward, whereby conflict is defined from 1 (low conflict) to 5 (high conflict), with a probability of .05 of choosing the LL reward as maximum conflict, and

(ii) the trial-wise drift rate, as derived from the DDM.

Further, extending our planned analyses, we assessed the relationship between response vigour and the subjective value differences based on the estimated parameters of the best-fitting drift DDM.

\subsubsection{Measures of response vigour}

Handgrip force

To examine the relationship between the characteristics of the handgrip response and the choice behaviour and estimated model parameters (subjective value differences, choice probabilities and decision conflict), we modelled the handgrip response on individual trials with a Gaussian function of the form

$$
f(x)=a e^{-\left(\frac{x-b}{c}\right)^{2}}+d
$$

using MATLAB's fit function, where the coefficient $a$ is the amplitude (height of peak), $b$ the centroid (centre of peak), $c$ the width (width of peak) and $d$ is a constant (to model offsets from zero). The handgrip data were fitted trial-wise per participant. To test for a magnitude effect in the gripforce response, we used frequentist significance tests (one-tailed for amplitude and centroid, see hypothesis iv [section 1], significance threshold set at .05, not corrected for multiple comparisons).

\section{Gaze shifting}

Using the eye tracking data, we assessed the relationship between the frequency of gaze shifts between the choice options and the associated decision conflict (see section 2.4.2). We defined fixation shifts as the number of switches between the left and right option (skipping middle fixations, see also section 2.2).

\subsubsection{Relationship between value difference, conflict and response vigour}

To assess the relationship between conflict and response vigour, we regressed response vigour (single-trial Gaussian grip force model parameters and number of fixation shifts) onto the response conflict measures. We fitted a hierarchical Bayesian linear regression of the form

$$
y_{t}=\alpha+\beta_{1} * a_{t}+\beta_{2} * b_{t}+\beta_{3} * c_{t}+\beta_{4} * d_{t}
$$

where $y$ is the conflict on trial $t$, operationalised either (1) based on the choice probabilites from the softmax model (see section 2.4.2), (2) as the trial-wise drift rate, based on the estimated parameters 
of the best fitting drift diffusion model, or (3) as the value difference between the (discounted) LL and SS reward on trial $t$, based on the estimated subject-specific $k$ parameters of the DDM (see Equation 1).

Since we observed no relationship between response vigour and conflict, neither for choice probability (softmax model) nor trial-wise drift rate (DDM), we extended our analyses plan and also regressed response vigour on the (absolute) subjective value differences. We reasoned that this might be attributable to the fact that both predictors are insensitive to increasingly higher value differences: in the softmax model, these are mapped to a conflict of 0 , whereas in the DDM these are mapped to a maximum drift rate of $v_{\max }$. The estimated grip force parameters $a, b$, and $c$, and the number of fixation shifts were within-subjects $z$-standardised before entering the regression. The parameter $d$ corresponds to the absolute number of fixation shifts between the options. Since we exluded each participant's slowest and fastest $2.5 \%$ of trials within the scope of the drift diffusion model (see section 2.4.2), the respective trials were likewise removed from the gripforce and gaze data.

We report Bayes factors (BFs) for directional effects (Kass \& Raftery, 1995) for the $\beta$ - hyperparameters, via kernel density estimation in MATLAB (The MathWorks, Inc., version R2019a). The Bayes factors are defined as the ratio of the integral of the posterior distribution from - $\infty$ to 0 versus the integral from 0 to $\infty$. We consider BFs between 1 and 3 as anecdotal evidence, BFs between 3 and 10 as moderate evidence, BFs between 10 and 30 as strong evidence, BFs between 30 and 100 as very strong evidence, and BFs above 100 as extreme evidence for the H1. The inverse of these values reflect the corresponding evidence for the H0 (Beard, Dienes, Muirhead, \& West, 2016; Lee \& Wagenmakers, 2013). We further report the posterior highest density intervals (HDI) along with the regions of practical equivalence (ROPE, limits for $\beta= \pm 0.05$ as for standardised variables) (Kruschke, 2018) for the posterior distributions of the regression coefficients.

\subsubsection{Parameter estimation and model comparison}

The parameter distributions of the softmax, drift diffusion and regression models were estimated through Markov chain Monte Carlo (MCMC) simulation as implemented in JAGS (Plummer, 2003, version 4.3.0), using MATLAB (The MathWorks, Inc., version R2019a) and the MATJAGS inferface for JAGS (Steyvers, 2018, version 1.3.2). We implemented a hierarchical Bayesian framework, in which the parameters for each subject are drawn from group-level gaussian distributions. We ran two chains with a burn-in period of 50,000 samples and thinning of two. We determined chain convergence of the chains such that $\hat{R} \leq 1.01$ (Gelman, Rubin, et al., 1992). For comparing the variants of the drift diffusion models, we ranked them according to the deviance information criterion (Spiegelhalter, Best, Carlin, \& Van Der Linde, 2002, DIC).

\subsubsection{Posterior predictive response time distributions}

To ensure that the best-fitting model reflects and reproduces the observed data, we simulated 10,000 datasets based on the posterior distributions of the respective hierarchical model. For each individual participant, the model-predicted RT distributions were smoothed with a kernel 
Response vigour during intertemporal choice

smoothing function using density estimation (using MATLAB's ksdensity function) and overlaid onto the observed RT distributions.

\section{RESULTS}

\subsection{Model-free analyses}

The participants made significantly more LL selections in the high $(M=63.00, S D=19.28)$ as compared to the low $(M=53.30, S D=21.09)$ magnitude condition $(t(39)=-10.12, p<.001$, onetailed), reflecting the predicted magnitude effect. However, such a magnitude effect was not present in the response time patterns. The mean RTs were not significantly different between the low $(M=3.03, S D=0.69)$ and high $(M=3.03, S D=0.67)$ condition $(t(39)=-0.01, p=.498$, one-tailed).

\subsection{Softmax choice rule}

We modelled the choices using the softmax choice rule, using both the absolute and normalised reward values. As hypothesised, we found a magnitude effect for temporal discounting, indicated by the negative shift parameter $s_{\log (k)}$, which models the change in $\log (k)$ from the low to the high condition (see Table 1). We observed a close correspondence of the parameter estimates from the softmax model based on absolute vs. normalised values, except for $\beta$ (see Figure 1), which scales with the value differences (see Equation 2).
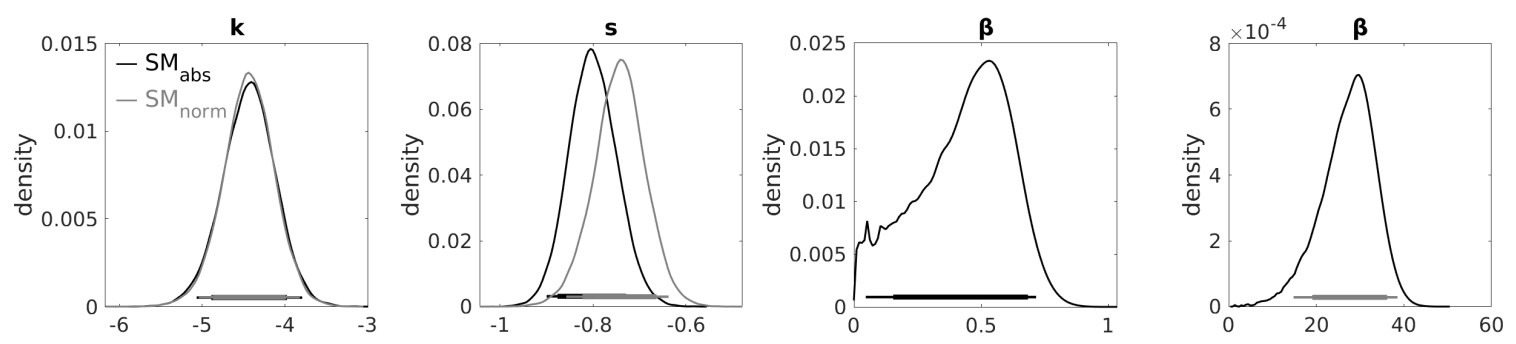

Figure 1: Posterior distributions of the group-level parameter means from the softmax models based on absolute $\left(\mathrm{SM}_{\mathrm{abs}}\right)$ and normalised $\left(\mathrm{SM}_{\text {norm }}\right)$ values. $\log (k)$ : discounting parameter, $s$ : shift in $\log (k), \beta$ : temperature parameter. Horizontal solid lines indicate the $85 \%$ and $95 \%$ highest density interval. 


\subsection{Drift diffusion modelling}

\subsubsection{Model comparison}

We compared the fit of different variants of the DDM, including models with a linear $\left(\mathrm{DDM}_{\mathrm{lin}}\right)$ and non-linear scaling $\left(\mathrm{DDM}_{\mathrm{sig}}\right.$, and $\left.\mathrm{DDM}_{\text {sig-shift }}\right)$ of the drift rate by the subjective value differences, and a model including parameters to model changes in the parameter distributions from the low to high condition $\left(\mathrm{DDM}_{\text {sig-shift }}\right)$. As a baseline comparison, we formulated a model comprising no value modulation (constant drift rate, $\mathrm{DDM}_{0}$ ). Further, we assessed the fit of all models using absolute vs. normalised values (see section 2.4.2). The models implementing a non-linear scaling of the drift rate provided a superior fit to the data compared to models with a linear scaling. This was true for models operating on absolute and normalised values. Also, both the linear and non-linear models provided a superior fit compared to the $\mathrm{DDM}_{0}$, see Table 2 and 3.

Table 2: Model comparison of the variants of the drift diffusion models of temporal discounting using absolute values.

\begin{tabular}{lllll}
\hline & Value scaling & Value function & DIC & Rank \\
\hline $\mathbf{D D M}_{\mathbf{0}}$ & - & - & 26830 & 4 \\
$\mathbf{D D M}_{\text {lin }}$ & Linear & Hyperbolic & 24769 & 3 \\
$\mathbf{D D M}_{\text {sig }}$ & Sigmoid & Hyperbolic + shift & 22213 & 2 \\
$\mathbf{D D M}_{\text {sig-shift }}$ & Sigmoid & Hyperbolic + shift & 22179 & 1 \\
\hline
\end{tabular}

Note. DIC = deviance information criterion; 0: no value scaling of the drift rate; lin: linear value scaling of the drift rate; sig: sigmoid value scaling of the drift rate. The $\mathrm{DDM}_{\text {sig-shift }}$ includes additional shift parameters for $\alpha, \tau, z, v, v_{\text {coeff }}$, and $v_{\max }$ to models changes from the low to high condition.

Table 3: Model comparison of the variants of the drift diffusion models of temporal discounting using normalised values.

\begin{tabular}{lllll}
\hline & Value scaling & Value function & DIC & Rank \\
\hline $\mathbf{D D M}_{\mathbf{0}}$ & - & - & 26830 & 4 \\
$\mathbf{D D M}_{\text {lin }}$ & Linear & Hyperbolic & 24286 & 3 \\
$\mathbf{D D M}_{\text {sig }}$ & Sigmoid & Hyperbolic + shift & 22210 & 2 \\
$\mathbf{D D M}_{\text {sig-shift }}$ & Sigmoid & Hyperbolic + shift & 22170 & 1 \\
\hline
\end{tabular}

Note. DIC = deviance information criterion; 0 : no value scaling of the drift rate; lin: linear value scaling of the drift rate; sig: sigmoid value scaling of the drift rate. The $\mathrm{DDM}_{\text {sig-shift }}$ includes additional shift parameters for $\alpha, \tau, z, v, v_{\text {coeff }}$, and $v_{\max }$ to models changes from the low to high condition.

\subsubsection{Posterior predictive response time distributions}

To verify that the best-fitting model can reproduce the observed RT distributions, we examined the posterior predictive RT distributions per participant (see section 2.4.6). The posterior predictive RT 
Response vigour during intertemporal choice

distributions of the $\mathrm{DDM}_{\text {sig-shift }}$ (using normalised values), along with the observed response time distributions, are depicted in Figure 2 (see Figure S1 for the posterior predictive response time distributions of the $\mathrm{DDM}_{\text {sig-shift }}$ using absolute values). The comparison showed that the model captures the characteristics of the response time distributions well.
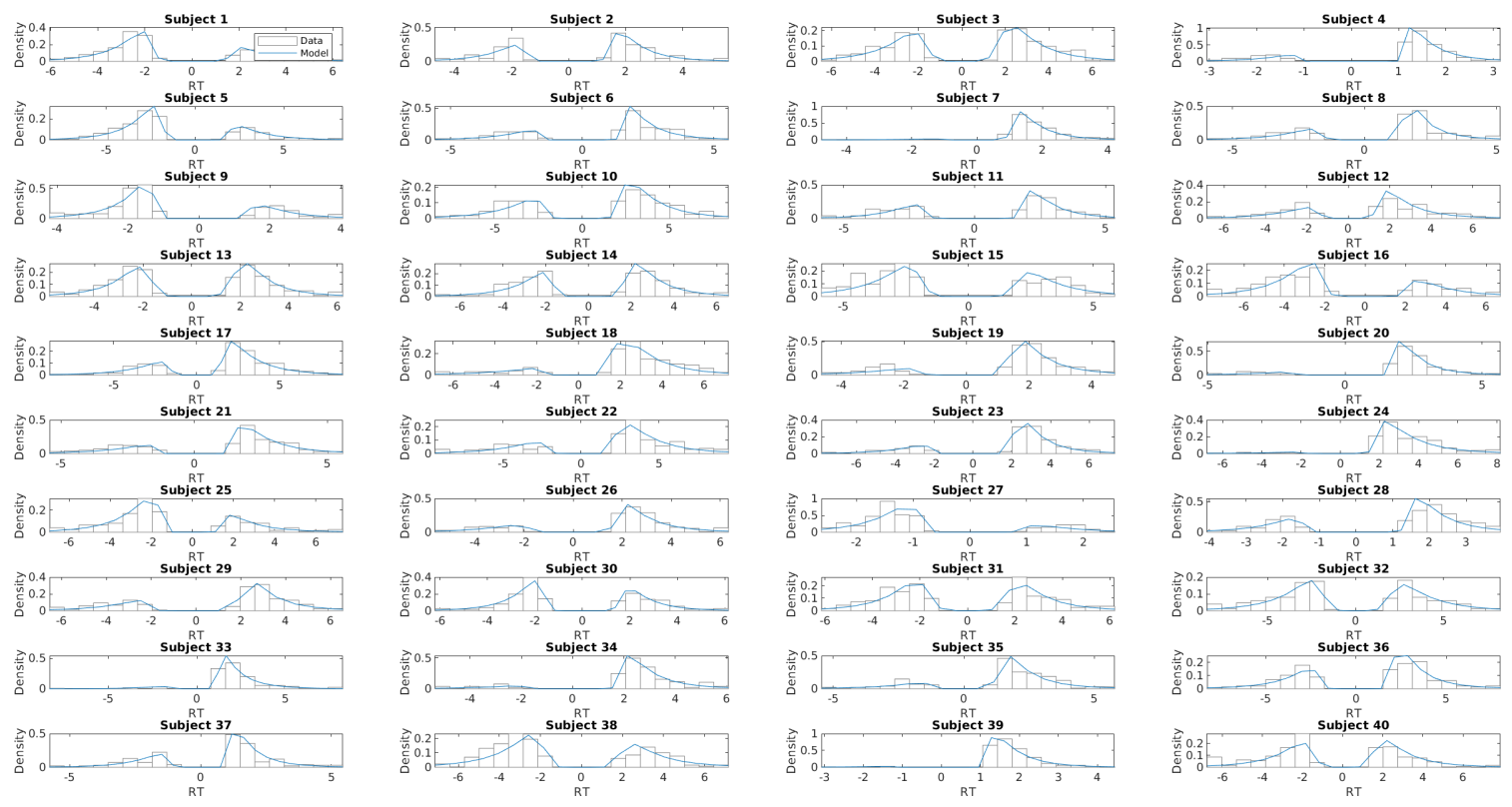

Figure 2: Posterior predictive response time distributions of the $\mathrm{DDM}_{\text {sig-shift }}$ (using normalised values) for each participant, overlaid on the histograms of the observed RT distributions.

\subsubsection{Analysis of model parameters}

In general, the estimated non-decision times $\tau$ were longer than in typical laboratory experimental setups (> $1000 \mathrm{~ms}$ ), comparable to the estimated non-decision times from a recent study using a VR environment, where the participants logged their responses using VR-compatible controllers, as opposed to simple response keys (Bruder, Scharer, \& Peters, 2021). This is likely due to the task's requirement of preselecting an option through visual fixation before finally selecting it using the hand dynamometer. Further, we observed a positive association between the value differences and trial-wise drift rates, as indicated by the consistently positive drift rate coefficient parameter $v_{\text {coeff }}$ (see Table 4 and 5).

\section{Magnitude effects on model parameters}

For all models with value modulation of the drift rate, we observed an effect of reward magnitude on $s_{\log (k)}$ (see Table 4), reflecting reduced discounting in the high compared to the low magnitude condition. This was also true for the models operating on normalised values (see Table 5). The starting point parameter $z$ was close to .5 , indicating no strong bias towards either decision bound- 
ary, (SS rewards), with a rather small shift towards the upper boundary in the high magnitude condition. The effects of reward magnitude on the other parameters were negligible.

\section{Effects of value normalisation}

Comparing the models based on absolute vs. normalised values, we observed a good correspondence of all model parameters, with the exception of $v_{\text {coeff }}$ and $v_{c o e f f_{\text {shift }}}$, which of course scale directly with value differences.

Table 4: Parameter group means and 95\% HDIs of the posterior distributions of the drift diffusion models using absolute values.

\begin{tabular}{lllll}
\hline & DDM $_{\mathbf{0}}$ & $\mathbf{D D M}_{\text {lin }}$ & DDM $_{\text {sig }}$ & DDM $_{\text {sig-shift }}$ \\
\hline$\alpha$ & $2.72(2.59$ to 2.86$)$ & $2.90(2.76$ to 3.04$)$ & $3.27(3.09$ to 3.43$)$ & $3.23(3.05$ to 3.41$)$ \\
$s_{\alpha}$ & - & - & - & $0.09(-0.01$ to 0.19$)$ \\
$\tau$ & $1.30(1.22$ to 1.39$)$ & $1.30(1.23$ to 1.39$)$ & $1.23(1.15$ to 1.31$)$ & $1.23(1.15$ to 1.31$)$ \\
$s_{\tau}$ & - & - & - & $0.01(-0.01$ to 0.04$)$ \\
$z$ & $0.53(0.52$ to 0.55$)$ & $0.53(0.51$ to 0.56$)$ & $0.51(0.49$ to 0.52$)$ & $0.50(0.49$ to 0.52$)$ \\
$s_{z}$ & - & - & - & $0.02(0.00$ to 0.03$)$ \\
$v$ & $0.18(0.04$ to 0.31$)$ & - & - & - \\
$v_{\text {coeff }}$ & - & $0.05(0.04$ to 0.05$)$ & $0.77(0.61$ to 0.94$)$ & $0.78(0.62$ to 0.95$)$ \\
$s_{v_{\text {coeff }}}$ & - & - & - & $-0.08(-0.17$ to 0.01$)$ \\
$v_{\max }$ & - & - & $1.07(0.98$ to 1.16$)$ & $1.10(1.01$ to 1.19$)$ \\
$s_{v_{\max }}$ & - & - & - & $-0.04(-0.11$ to 0.03$)$ \\
$\log (k)$ & - & $-4.42(-5.08$ to -3.78$)$ & $-4.45(-5.07$ to -3.83$)$ & $-4.47(-5.07$ to -3.85$)$ \\
$s_{\log (k)}$ & - & $-0.52(-0.71$ to -0.34$)$ & $-0.82(-0.93$ to -0.71$)$ & $-0.77(-0.89$ to -0.65$)$ \\
\hline
\end{tabular}

Note. HDI: highest density interval; 0: no value scaling of the drift rate; lin: linear value scaling of the drift rate; sig: sigmoid value scaling of the drift rate; s: shift parameter for the changes in parameter value from the low to high magnitude condition. 
Response vigour during intertemporal choice

Table 5: Parameter group means and 95\% HDIs of the posterior distributions of the drift diffusion models using normalised values.

\begin{tabular}{|c|c|c|c|c|}
\hline & $\mathrm{DDM}_{0}$ & $\mathrm{DDM}_{\text {lin }}$ & $\mathrm{DDM}_{\text {sig }}$ & DDM $_{\text {sig-shift }}$ \\
\hline$\alpha$ & 2.72 (2.59 to 2.86$)$ & 2.95 (2.81 to 3.09$)$ & 3.27 (3.10 to 3.45$)$ & 3.24 (3.05 to 3.41$)$ \\
\hline$s_{\alpha}$ & - & - & - & $0.07(-0.03$ to 0.18$)$ \\
\hline$\tau$ & 1.30 (1.22 to 1.39$)$ & 1.30 (1.22 to 1.38$)$ & $1.23(1.15$ to 1.31$)$ & 1.23 (1.15 to 1.31$)$ \\
\hline$s_{\tau}$ & - & - & - & $0.02(-0.01$ to 0.04$)$ \\
\hline$z$ & 0.53 (0.52 to 0.55$)$ & $0.53(0.51$ to 0.56$)$ & $0.51(0.50$ to 0.52$)$ & $0.50(0.49$ to 0.52$)$ \\
\hline$s_{z}$ & - & - & - & $0.02(0.00$ to 0.03$)$ \\
\hline$v$ & $0.18(0.04$ to 0.31$)$ & - & - & - \\
\hline$v_{\text {coeff }}$ & - & $3.00(2.68$ to 3.32$)$ & 39.44 (31.68 to 47.92$)$ & $38.11(30.11$ to 46.90$)$ \\
\hline$s_{v_{c o e f f}}$ & - & - & - & $2.58(-0.24$ to 4.40$)$ \\
\hline$v_{\max }$ & - & - & 1.07 (0.99 to 1.16$)$ & 1.05 (0.96 to 1.14$)$ \\
\hline$s_{v_{\max }}$ & - & - & - & $0.04(-0.02$ to 0.10$)$ \\
\hline $\log (k)$ & - & $-4.19(-4.78$ to -3.60$)$ & $-4.48(-5.13$ to -3.87$)$ & $-4.49(-5.14$ to -3.88$)$ \\
\hline$s_{\log (k)}$ & - & $-0.75(-0.90$ to -0.60$)$ & $-0.79(-0.92$ to -0.68$)$ & $-0.76(-0.89$ to -0.64$)$ \\
\hline
\end{tabular}

Note. HDI: highest density interval; 0: no value scaling of the drift rate; lin: linear value scaling of the drift rate; sig: sigmoid value scaling of the drift rate; s: shift parameter for the changes in parameter value from the low to high magnitude condition.

\subsection{Response vigour}

The grip force responses were modelled with a Gaussian function (see Figure 3, 1 term plus constant, mean (range) goodness-of-fit across all trials and participants: R-squared $=0.98(0.29$ $1.00)$, adjusted R-squared $=0.98(0.29-1.00)$, root-mean-square error $=0.004(0.0002-0.16)$. The parameter means (amplitude, centroid and width) per condition (low, high) are listed in Table 6, for mean values per participant and condition see Figure S2.

Since the data were non-normal (as assessed with Lilliefors tests yielding $p<.001$ for all tests), we performed Wilcoxon signed-rank tests to check for parameter differences between the low and high condition. In line with our preregistered hypothesis, the amplitude was significantly higher for the high compared to the low condition $(z=1.90, p=.029$, one-tailed). In contrast to our preregistered hypothesis, the centroid, and also the width, did not differ between conditions $(z$ $=0.73, p=.768$, one-tailed, and $z=1.75, p=.081$, two-tailed). Within-subject differences of the parameters between the two conditions are depicted in Figure S3. 
Response vigour during intertemporal choice

Table 6: Parameters of the gaussian-modelled grip force responses (means and standard deviations).

\begin{tabular}{lll}
\hline & Low condition & High condition \\
\hline Amplitude & $0.2142(0.1414)$ & $0.2179(0.1448)$ \\
Centroid & $6294.04(3071.99)$ & $6302.33(3046.76)$ \\
Width & $265.66(98.03)$ & $267.49(98.36)$ \\
\hline
\end{tabular}

Note. Amplitude has been normalised to MVC (maximal voluntary contraction).
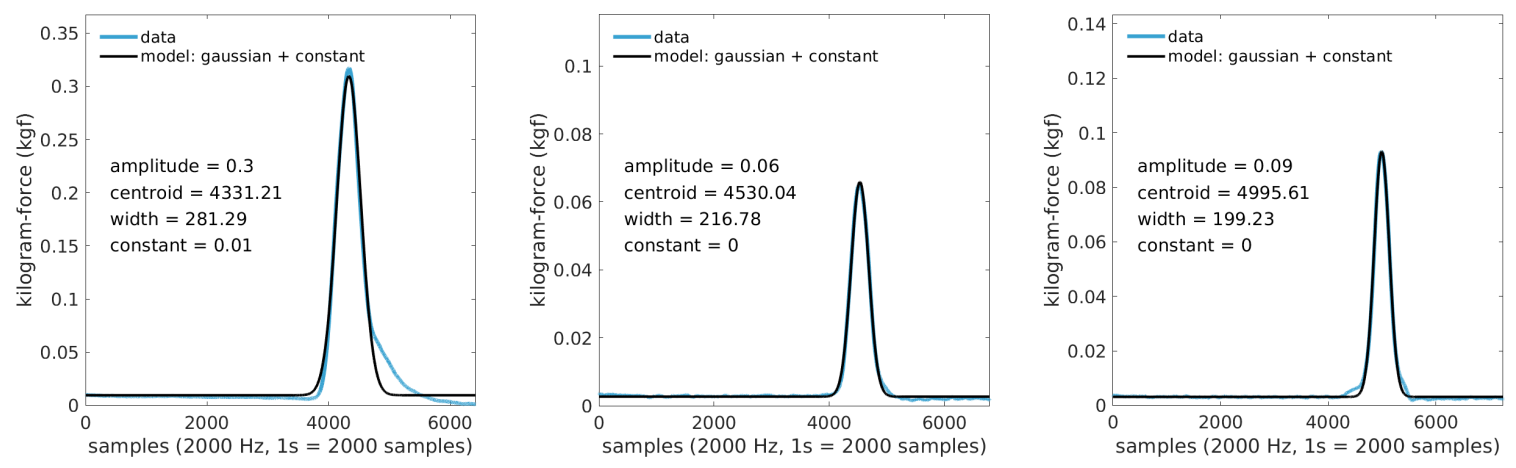

Figure 3: Modelled grip response of three trials $(14,152$ and 180) from one participant.

\subsubsection{Decision conflict and response vigour}

Conflict based on choice probability (softmax model)

Our first operationalisation of response conflict was based on the softmax choice probabilities. Because condition effects are more straightforward to interpret in the normalised model (see section 3.2), the following analyses are based on this model. The mean values for amplitude, centroid and number of gaze shifts for trials of a given response conflict (binned from 1 to 5) are depicted participant-wise in Figure 4 and listed in Table 7. The Bayesian regression is based on a continuous conflict measure (probabilities $>.5$ are 'flipped' to provide a common scaling from low to high conflict, whereby .5 represents the maximum conflict). The posterior distributions of the group-level parameter means for the regression coefficients are depicted in Figure 5 (medians: $\alpha=$ 0.12 [intercept] $\beta_{1}=-0.01$ [amplitude], $\beta_{2}=0.02$ [centroid], $\beta_{3}=-0.004$ [width], $\beta_{4}=0.001$ [ $N$ gaze shifts]). 
Response vigour during intertemporal choice

Table 7: Mean amplitude, centroid and number of gaze shifts per conflict bin.

\begin{tabular}{llllll}
\hline & $\mathbf{1}$ & $\mathbf{2}$ & $\mathbf{3}$ & $\mathbf{4}$ & $\mathbf{5}$ \\
\hline Amplitude & 0.2188 & 0.2128 & 0.2102 & 0.2135 & 0.2137 \\
Centroid & 5885.39 & 6536.03 & 6412.59 & 6557.73 & 6632.03 \\
Gaze shifts & 2.79 & 3.52 & 3.05 & 3.17 & 3.42 \\
\hline
\end{tabular}

Note. Conflict is defined from 1 (low conflict) to 5 (high conflict), with a probability of .5 of choosing the LL reward as maximum conflict. Amplitude has been normalised to MVC (maximal voluntary contraction).
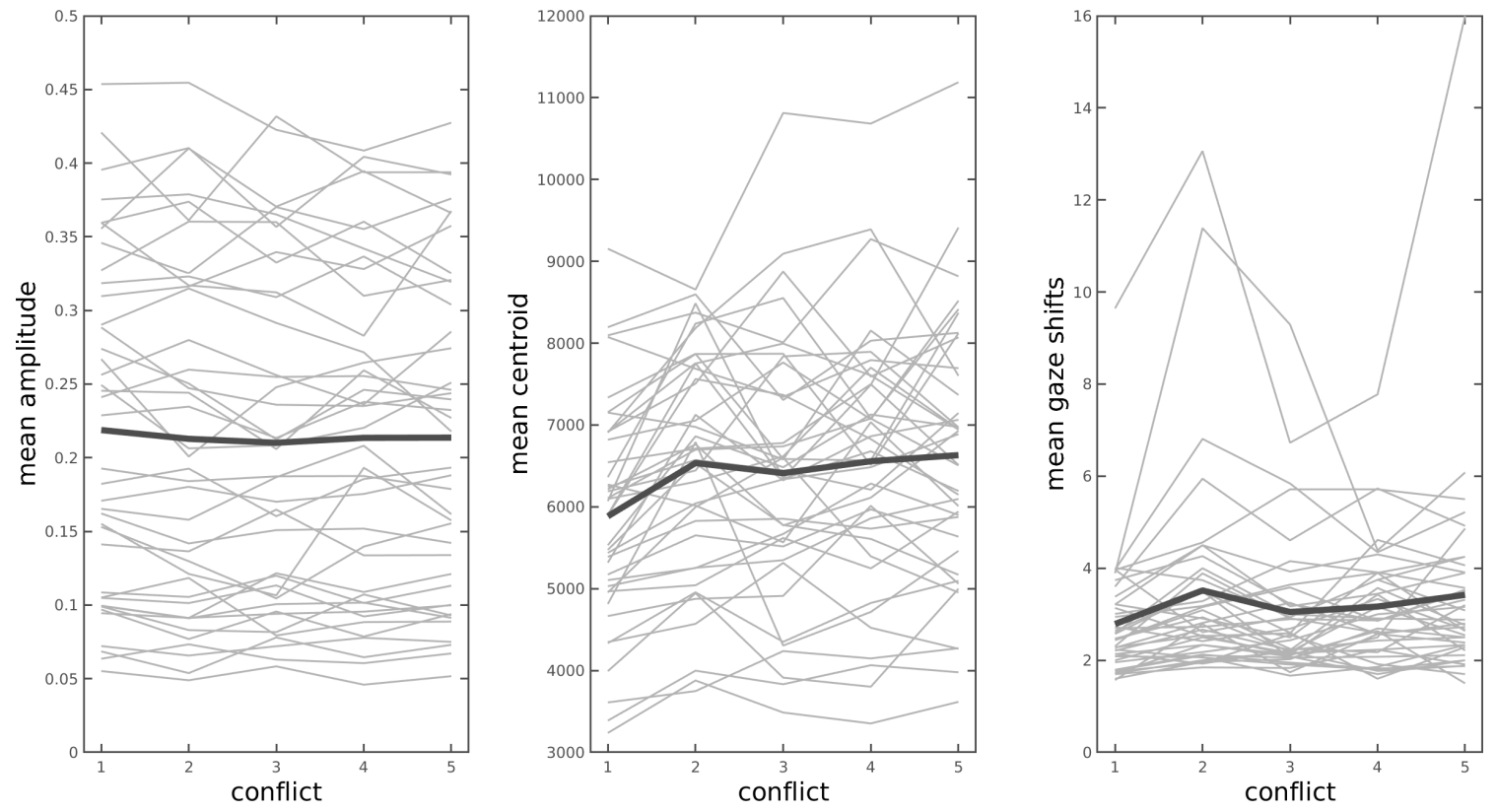

Figure 4: Mean amplitude and centroid of the Gaussian-modelled grip force response and mean number of gaze shifts (from SS to LL, and vice versa) for trials of a given (binned) response conflict for each participant. Thick lines depict the mean values across participants. Conflict is defined from 1 (low conflict) to 5 (high conflict), with a probability of .5 of choosing the LL reward as maximum conflict. 

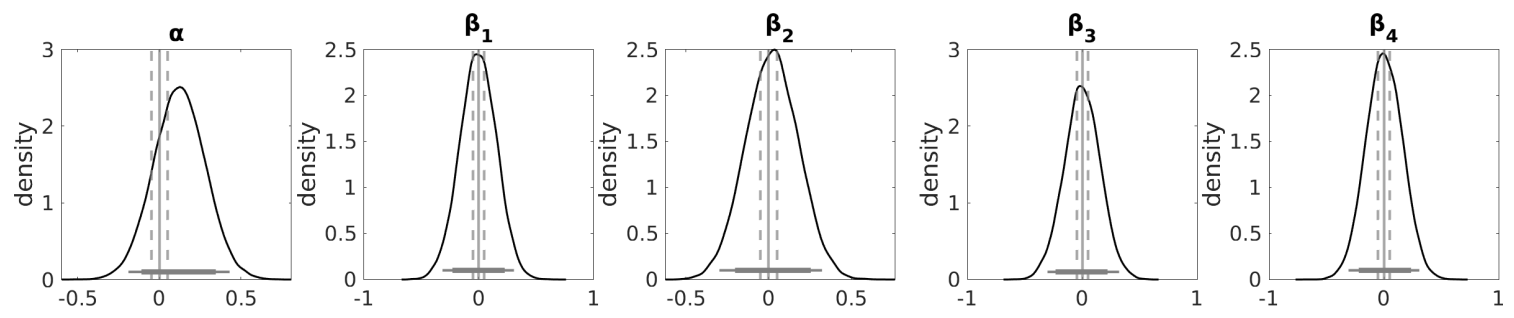

Figure 5: Hierarchical Bayesian regression results. Regression of the parameters of the Gaussianmodelled grip force response onto the trial-wise response conflict based on the choice probabilities (softmax model). Posterior distributions of the group-level parameter means. $\alpha$ : intercept, $\beta 1$ : coefficient for amplitude, $\beta_{2}$ : coefficient for centroid, $\beta_{3}$ : coefficient for width, $\beta_{4}$ : coefficient for fixation shift. Horizontal solid lines indicate the $85 \%$ and $95 \%$ highest density interval. Vertical solid lines indicate $x=0$, and vertical dashed lines indicate the lower and upper bounds of the region of practical equivalence (ROPE).

The Bayes factors for the regression coefficients for amplitude, centroid, and width of the grip response, and for the numbers of fixation shifts provide only anecdotal evidence for values greater than zero vs. smaller than zero (BF for $\beta_{1}: 0.95, \mathrm{BF}$ for $\beta_{2}: 1.25, \mathrm{BF}$ for $\beta_{3}: 0.97, \mathrm{BF}$ for $\beta_{4}: 1.09$ ). Since the $95 \%$ HDIs of all the posterior distributions fall neither completely inside nor outside the $\mathrm{ROPE}$, we remain undecided for all three $\beta$ regression coefficients.

\section{Conflict based on subjective value differences (DDM)}

The second operationalisation of response conflict was based on the trial-wise drift rate calculated based on the estimated parameters of the highest-ranked DDM using normalised values $\left(\mathrm{DDM}_{\text {sig-shift }}\right)$. Since we found no evidence that any of the regression coefficients for response vigour were greater than vs. smaller than zero (or vice versa), we refer the reader to section S1 of the supplementary material.

Finally, we regressed the estimated grip force parameters amplitude, centroid and width, and the number of fixation shifts onto the subjective value differences between the (discounted) LL and SS rewards, based on the subject-specific $k$ parameters of the highest-ranked model using absolute values $\left(\mathrm{DDM}_{\text {sig-shift }}\right)$. Recall that the analyses of the magnitude effect yielded an effect of condition, i.e. higher grip force amplitudes in the high compared to the low condition (see 3.4). Because condition differences in reward magnitudes are eliminated in the DDM based on normalised values (section S2), the regression on subjective value differences is based on the DDM using absolute values.

The mean values for amplitude, centroid and number of gaze shifts for trials of a given value difference bin are depicted participant-wise in Figure 6. The posterior distributions of the group-level parameter means for the regression coefficients are depicted in Figure 7 . The medians of the group-level posterior distributions were as follows: $\alpha=3.33$ (intercept) $\beta_{1}=0.46$ (amplitude), $\beta_{2}=-1.20$ (centroid), $\beta_{3}=0.19$ (width), $\beta_{4}=-0.48$ ( $N$ gaze shifts). 

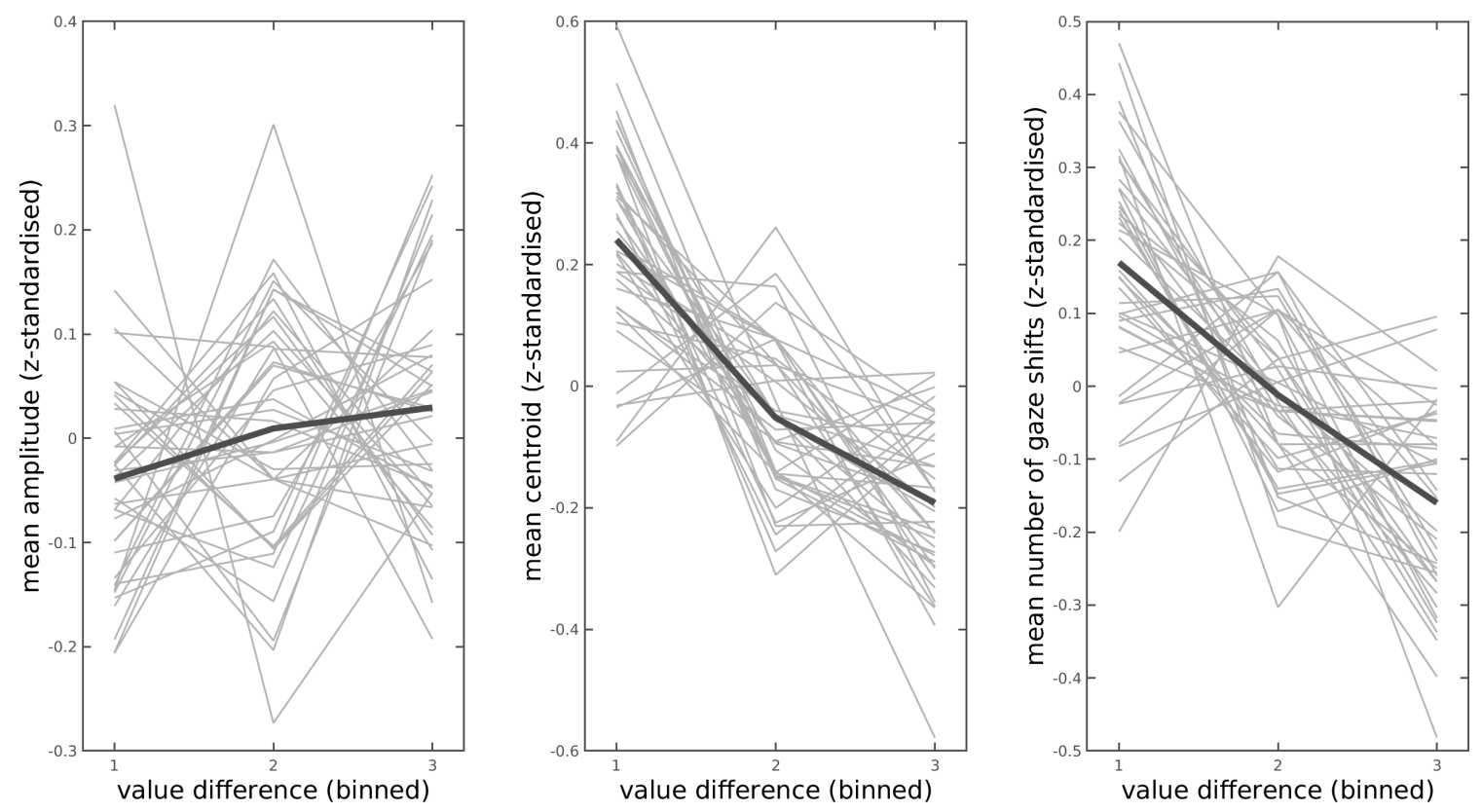

Figure 6: Mean amplitude and centroid of the Gaussian-modelled grip force response and mean number of gaze shifts (from SS to LL, and vice versa) for trials of a given value difference bin. The (absolute) value differences were $z$-standardised and binned participant-wise into 3 groups of equal size (based on quantile ranks of the values, 1: lower value differences, 3: higher value differences). Thick lines depict the mean values across participants.
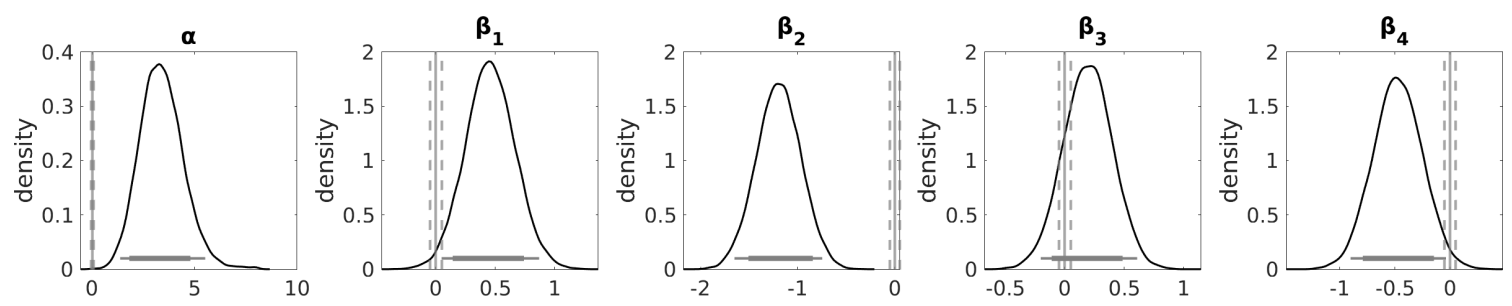

Figure 7: Hierarchical Bayesian regression results. Regression of the parameters of the Gaussianmodelled grip force response onto the value differences (DDM). Posterior distributions of the group-level parameter means. $\alpha$ : intercept, $\beta 1$ : coefficient for grip force amplitude, $\beta_{2}$ : coefficient for grip force centroid, $\beta_{3}$ : coefficient for grip force width, $\beta_{4}$ : coefficient for fixation shift. Horizontal solid lines indicate the $85 \%$ and $95 \%$ highest density interval. Vertical solid lines indicate $\mathrm{x}=0$, and vertical dashed lines indicate the lower and upper bounds of the region of practical equivalence (ROPE).

The Bayes factors provide very strong evidence that the coefficient for amplitude is greater than zero vs. smaller than zero ( $\mathrm{BF}$ for $\beta_{1}$ : 79.50), extreme evidence that the coefficient for centroid is below zero vs. above zero ( $\mathrm{BF}$ for $\beta_{2}:>-10^{308}$ ), moderate evidence that the regression coefficient for grip force width is greater than zero vs. smaller than zero ( $\mathrm{BF}$ for $\beta_{3}$ : 5.06), and very strong evidence that the coefficient for number of fixation shifts is smaller vs. greater than zero (BF for 
$\beta_{4}:$ 69.61). For $\beta_{3}$ we remain undecided, since the $95 \%$ HDI of the posterior distribution is neither completely inside nor outside the ROPE. For $\beta_{2}$ we reject the null value (95\% HDI of posterior distribution entirely outside ROPE). For $\beta_{1}$ and $\beta_{4}$ we also reject the null value, since the $95 \%$ HDIs do not include zero and only $0.23 \%$ and $1.41 \%$ of the $95 \%$ HDI, respectively, overlap with the ROPE. This indicates higher grip force amplitudes, faster response times and a lower number of fixation shifts for trials with higher subjective value differences between the options.

\section{Discussion}

We explored whether value computation and response conflicts during intertemporal choice are reflected in measures of response vigour. For this purpose, we measured the handgrip force applied during choice, and the concurrent gaze shift patterns between the choice options. Assuming hyperbolic discounting, we compared variants of the softmax and drift diffusion model and assessed the relationship between the estimated model parameters and response vigour. The intertemporal choice task comprised two conditions, a low and high magnitude condition (low vs. high SS reward), which allowed us to directly assess the impact of overall smaller vs. larger reward magnitudes on response vigour. To represent both conditions in a single model, we included shift parameters to model the changes in parameter values from the low to the high magnitude condition.

We compared models with a linear and non-linear (sigmoid) modulation of the drift rate by the subjective value differences, and, since the drift rate parameter is dependent on the absolute magnitude of the option's values, models using absolute vs. normalised option values. We then analysed the relationship between decision conflict and response vigour, in particular the trial-wise amplitude, centroid and width of the Gaussian-modelled grip force response and the number of fixation shifts between the options. Further, we investigated if the magnitude effect, which describes reduced discounting for higher amounts (Ballard et al., 2017; Green et al., 1997), is also reflected in the grip force strength.

The choice and response time (RT) data were best accounted for by a DDM including a nonlinear modulation of the drift rate by the subjective value differences. As in previous studies, (Green et al., 1997; Wagner et al., 2020), we found a magnitude effect for temporal discounting, indicating that higher rewards were discounted less. This effect was also evident in response vigour: Higher forces were applied in the high vs. the low magnitude condition. In addition, trials with higher subjective value differences between the options were associated with higher grip forces, faster response times and a lower number of fixation shifts.

\section{Model comparison}

The choice and RT data were best accounted for by a drift diffusion model specifying a non-linear mapping between the subjective value differences and trial-wise drift rates. Following the DIC criterion, the variants of the DDMs implementing a transformation of the scaled value differences using a sigmoid function (Fontanesi et al., 2019) provided a superior fit to the data compared to both the DDM using a linear modulation and the DDM involving no value modulation. We found 
Response vigour during intertemporal choice

a close correspondence between the observed response time distributions and the response time distributions simulated using the estimated posterior parameter distributions, demonstrating that the best-fitting model captured the characteristics of the response time distributions reasonably well.

\section{Magnitude effect}

Replicating previous findings (Ballard et al., 2017; Green et al., 1997; Wagner et al., 2020), we found a magnitude effect for temporal discounting, such that higher rewards were discounted less. While the model-free analysis revealed more LL choices in the high compared to the low magnitude condition, the magnitude effect was further reflected in the $\log (k)_{\text {shift }}$ parameter, which was consistently negative in all variants of the softmax and drift diffusion models. Importantly, this magnitude effect was also reflected in response vigour: Looking at the amplitude parameter of the Gaussian-modelled gripforce response, we found that stronger forces were excerted in the high compared to the low magnitude condition. Contrary to our hypothesis, the RTs were not significantly different between the two conditions. The effect of reward magnitude on the discount rate (reduced discounting for higher rewards) appears to be a consistent effect (Ballard et al., 2017; Green et al., 1997; Wagner et al., 2020), and our data reveal that this effect is reflected in both choice behaviour and motor response vigour (grip force) during response selection.

\section{Decision conflict and response vigour}

First, we carried out a model-based analysis of the trial-wise grip force time courses. A gaussian model, decomposing grip force time courses into amplitude, centroid and width parameters for each trial provided an excellent fit to the single-trial grip force trajectories (mean R-squared $=.98$ ). We then analysed the relationship between decision conflict and the trial-wise response vigour measures, operationalising decision conflict based on the choice probability as derived from the softmax choice rule, and based on the trial-wise drift rate, as derived from the best-fitting DDM. Contrary to our hypothesis, however, we found no relationship between decision conflict and response vigour. However, regressing response vigour directly on the subjective value differences (based on the estimated parameters of the best-fitting DDM, we found that the amplitude and centroid of the grip response, as well as the number of fixation shifts were significantly related to these. With increasing subjective value differences between options, grip force amplitude increased, and RTs and the number of fixation shifts decreased.

The null effects for the conflict measure based on the softmax model likely arise because for large value differences, the conflict predictor approaches zero. The second regression was based on the drift diffusion model using a non-linear (sigmoid) scaling of the drift rate by the subjective value differences, so we speculate that the null effects for conflict based on the drift rate arise because the drift rate does not scale linearly with the value differences (as the value difference exceeds an individual threshold, the corresponding drift rate is mapped to $v_{\max }$ ). We therefore assume that the effects we find when regressing response vigour directly on the subjective value differences are driven by trials with large absolute value differences. Taken together, these results 
suggest that the observed associations between value differences and grip force parameters are driven by absolute value differences, rather than decision conflict.

This suggests that valuation or implicit motivation could be reflected in these measures. In contrast to Pessiglione and colleagues (2007), where the force produced was related to payout (reward height magnitude was presented subliminally), we kept the force produced unrelated to the payout. Therefore, even when the force produced is unrelated to the payout (and the participants are unaware that force production is being measured), it is nonetheless related to the subjective value difference between the options. Also, interestingly, although physical effort, similar to delay, is considered a decision cost (Klein-Flügge et al., 2015; Prévost et al., 2010), the participants nonetheless applied more force in trials with higher value differences. This leads us to the conclusion that motivational processes are reflected in response vigour.

\section{Dopamine and response vigour}

Although dopamine neurotransmission was not measured in the present study, the observed effects might be mediated by dopamine. For example, pharmalogical enhancement of dopamine transmission increases the willingness of animals to accept delays and to expend effort to obtain rewards (for a review, see Webber, Lopez-Gamundi, Stamatovich, de Wit, \& Wardle, 2020). Two studies with human subjects also reported higher force production in states with augmented dopamine transmission (Chong et al., 2015; Le Bouc et al., 2016; Michely et al., 2020). Further, augmented dopamine transmission increased response vigour (reduced reaction times) in a temporal discounting and reinforcement learning task (Rihet, Possamaï, Micallef-Roll, Blin, \& Hasbroucq, 2002; Westbrook et al., 2020; Wagner et al., 2020).

\section{Relevance}

Several maladaptive behaviours and psychiatric conditions, including impulsivity, substance use disorders and behavioural addictions, have been linked to increased discount rates (see, e.g. Karakula et al., 2016; Steward et al., 2017; Wiehler \& Peters, 2015; Yi et al., 2010). Our results suggest that in addition to choices and response times, measures of response vigour may provide information regarding valuation during intertemporal choice.

The integration of reward and effort involves dopaminergic computations (see, e.g. Michely et al., 2020). Congruously, effort-based decision-making appears to be disrupted in conditions related to dopaminergic function, such as major depressive disorder and Parkinson's disease (Le Bouc et al., 2016; Treadway, Bossaller, Shelton, \& Zald, 2012), underlining the relevance of measuring response vigour during value-based decision-making.

\section{Limitations}

Finally, there are some limitations to our study. Since the usage of a force transducer functions as a single key, some method of preselecting one of two options is necessary. Choice selection was thus implemented such that an option was preselected by visual fixation and selected by subsequently 
pressing the force transducer. Hence, an option could only be chosen if it was concurrently fixated, which may have restricted the gaze patterns.

Although, based on the literature, an involvement of dopamine in the effects examined here is likely, dopamine neurotransmission was neither measured nor manipulated. Future studies would benefit from examining this in greater detail.

\section{Conclusions}

In the present work, we investigated response vigour, specifically gaze shifting and grip force applied during response selection as an implicit measures of subjective utility during intertemporal choice. Comparing variants of the drift diffusion model, we found that the choices and response times were best accounted for by a drift diffusion model that included a non-linear scaling of the drift rate by the subjective value differences. A magnitude effect for temporal discounting was apparent in both choice and response vigour, such that higher rewards were discounted less and selected with higher grip force. Further, the peak forces (grip force amplitudes), response times (grip force centroids) and the number of fixation shifts were related to the subjective value differences between the options. Normalising the options' values across conditions eliminated these effects. We conclude that the effects were likely driven by large absolute value differences between the options. The force applied was unrelated to the payout and the participants were not informed that force production was measured. Nonetheless, it was related to the subjective value differences between the options, suggesting that valuation or implicit motivation is reflected in response vigour. Future studies might explore the extend to which neuropsychiatric disorders associated with impairments in decision-making and effort are likewise associated with changes in such implicit measures of motivation.

\section{Code availability}

All model code is available on the Open Science Framework at https: / osf . io/agqsp/ (DOI: 10.17605/OSF.IO/AGQSP).

\section{DATA AVAILABILITY}

The data cannot be shared publicly, since the participants did not provide consent to public data access. The data are available at https://zenodo.org/record/5645885 (DOI: 10.5281/zenodo.5645885) for researchers who meet the criteria for access to confidential data.

\section{ACKNOWLEDGEMENTS}

This work was funded by the DFG (grant PE1627/5-1). 


\section{REFERENCES}

Ballard, I. C., Kim, B., Liatsis, A., Aydogan, G., Cohen, J. D., \& McClure, S. M. (2017). More is meaningful: the magnitude effect in intertemporal choice depends on self-control. Psychological science, 28(10), 1443-1454.

Beard, E., Dienes, Z., Muirhead, C., \& West, R. (2016). Using bayes factors for testing hypotheses about intervention effectiveness in addictions research. Addiction, 111(12), 2230-2247.

Bruder, L. R., Scharer, L., \& Peters, J. (2021). Reliability assessment of temporal discounting measures in virtual reality environments. Scientific reports, 11(1), 1-16.

Chib, V. S., Rangel, A., Shimojo, S., \& O’Doherty, J. P. (2009). Evidence for a common representation of decision values for dissimilar goods in human ventromedial prefrontal cortex. Journal of Neuroscience, 29(39), 12315-12320.

Chong, T. T.-J., Apps, M., Giehl, K., Sillence, A., Grima, L. L., \& Husain, M. (2017). Neurocomputational mechanisms underlying subjective valuation of effort costs. PLoS biology, 15(2), e1002598.

Chong, T. T.-J., Bonnelle, V., Manohar, S., Veromann, K.-R., Muhammed, K., Tofaris, G. K., ... Husain, M. (2015). Dopamine enhances willingness to exert effort for reward in parkinson's disease. cortex, 69, 40-46.

Fontanesi, L., Gluth, S., Spektor, M. S., \& Rieskamp, J. (2019). A reinforcement learning diffusion decision model for value-based decisions. Psychonomic bulletin \& review, 26(4), 1099-1121.

Gelman, A., Rubin, D. B., et al. (1992). Inference from iterative simulation using multiple sequences. Statistical science, 7(4), 457-472.

Green, L., Myerson, J., \& Macaux, E. W. (2005). Temporal discounting when the choice is between two delayed rewards. Journal of Experimental Psychology: Learning, Memory, and Cognition, 31(5), 1121.

Green, L., Myerson, J., \& McFadden, E. (1997). Rate of temporal discounting decreases with amount of reward. Memory \& cognition, 25(5), 715-723.

Kable, J. W., \& Glimcher, P. W. (2007). The neural correlates of subjective value during intertemporal choice. Nature neuroscience, 10(12), 1625-1633.

Karakula, S. L., Weiss, R. D., Griffin, M. L., Borges, A. M., Bailey, A. J., \& McHugh, R. K. (2016). Delay discounting in opioid use disorder: differences between heroin and prescription opioid users. Drug and alcohol dependence, 169, 68-72.

Kass, R. E., \& Raftery, A. E. (1995). Bayes factors. Journal of the american statistical association, 90(430), 773-795.

Klein-Flügge, M. C., Kennerley, S. W., Saraiva, A. C., Penny, W. D., \& Bestmann, S. (2015). Behavioral modeling of human choices reveals dissociable effects of physical effort and temporal delay on reward devaluation. PLoS Comput Biol, 11(3), e1004116.

Krajbich, I., Armel, C., \& Rangel, A. (2010). Visual fixations and the computation and comparison of value in simple choice. Nature neuroscience, 13(10), 1292-1298.

Kruschke, J. K. (2018). Rejecting or accepting parameter values in bayesian estimation. Advances in Methods and Practices in Psychological Science, 1(2), 270-280. 
Le Bouc, R., \& Pessiglione, M. (2013). Imaging social motivation: distinct brain mechanisms drive effort production during collaboration versus competition. Journal of Neuroscience, 33(40), 15894-15902.

Le Bouc, R., Rigoux, L., Schmidt, L., Degos, B., Welter, M.-L., Vidailhet, M., .. Pessiglione, M. (2016). Computational dissection of dopamine motor and motivational functions in humans. Journal of Neuroscience, 36(25), 6623-6633.

Lee, M., \& Wagenmakers, E.-J. (2013). Bayesian data analysis for cognitive science: A practical course. New York, NY: Cambridge University Press.

Levy, D. J., \& Glimcher, P. W. (2011). Comparing apples and oranges: using reward-specific and reward-general subjective value representation in the brain. Journal of Neuroscience, 31(41), 14693-14707.

Mazur, J. E. (1987). An adjusting procedure for studying delayed reinforcement. Commons, ML.; Mazur, JE.; Nevin, JA, 55-73.

Michely, J., Viswanathan, S., Hauser, T. U., Delker, L., Dolan, R. J., \& Grefkes, C. (2020). The role of dopamine in dynamic effort-reward integration. Neuropsychopharmacology, 1-6.

Moreira, D., \& Barbosa, F. (2019). Delay discounting in impulsive behavior: A systematic review. European Psychologist.

Odum, A. L. (2011). Delay discounting: I'm a k, you're a k. Journal of the experimental analysis of behavior, 96(3), 427-439.

Pedersen, M. L., Frank, M. J., \& Biele, G. (2017). The drift diffusion model as the choice rule in reinforcement learning. Psychonomic bulletin \& review, 24(4), 1234-1251.

Pessiglione, M., Schmidt, L., Draganski, B., Kalisch, R., Lau, H., Dolan, R. J., \& Frith, C. D. (2007). How the brain translates money into force: a neuroimaging study of subliminal motivation. Science, 316(5826), 904-906.

Peters, J., \& Büchel, C. (2009). Overlapping and distinct neural systems code for subjective value during intertemporal and risky decision making. Journal of Neuroscience, 29(50), 15727-15734.

Peters, J., \& D'Esposito, M. (2020). The drift diffusion model as the choice rule in inter-temporal and risky choice: a case study in medial orbitofrontal cortex lesion patients and controls. PLOS Computational Biology, 16(4), e1007615.

Plummer, M. (2003). Jags: A program for analysis of bayesian graphical models using gibbs sampling. Proceedings of the 3rd international workshop on distributed statistical computing.

Prévost, C., Pessiglione, M., Météreau, E., Cléry-Melin, M.-L., \& Dreher, J.-C. (2010). Separate valuation subsystems for delay and effort decision costs. Journal of Neuroscience, 30(42), 14080-14090.

Ratcliff, R., \& McKoon, G. (2008). The diffusion decision model: theory and data for two-choice decision tasks. Neural computation, 20(4), 873-922.

Reppert, T. R., Lempert, K. M., Glimcher, P. W., \& Shadmehr, R. (2015). Modulation of saccade vigor during value-based decision making. Journal of Neuroscience, 35(46), 15369-15378. 
Rihet, P., Possamaï, C.-A., Micallef-Roll, J., Blin, O., \& Hasbroucq, T. (2002). Dopamine and human information processing: a reaction-time analysis of the effect of levodopa in healthy subjects. Psychopharmacology, 163(1), 62-67.

Rogers, R. D. (2011). The roles of dopamine and serotonin in decision making: evidence from pharmacological experiments in humans. Neuropsychopharmacology, 36(1), 114-132.

Schultz, W. (2010). Dopamine signals for reward value and risk: basic and recent data. Behavioral and brain functions, 6(1), 1-9.

Shadmehr, R., Reppert, T. R., Summerside, E. M., Yoon, T., \& Ahmed, A. A. (2019). Movement vigor as a reflection of subjective economic utility. Trends in neurosciences.

Shahar, N., Hauser, T. U., Moutoussis, M., Moran, R., Keramati, M., Consortium, N., \& Dolan, R. J. (2019). Improving the reliability of model-based decision-making estimates in the two-stage decision task with reaction-times and drift-diffusion modeling. PLoS computational biology, 15(2), e1006803.

Spiegelhalter, D. J., Best, N. G., Carlin, B. P., \& Van Der Linde, A. (2002). Bayesian measures of model complexity and fit. Journal of the royal statistical society: Series b (statistical methodology), 64(4), 583-639.

Steward, T., Mestre-Bach, G., Fernández-Aranda, F., Granero, R., Perales, J. C., Navas, J. F., ... others (2017). Delay discounting and impulsivity traits in young and older gambling disorder patients. Addictive Behaviors, 71, 96-103.

Sutton, R. S., \& Barto, A. G. (2018). Reinforcement learning: An introduction. MIT press.

Treadway, M. T., Bossaller, N. A., Shelton, R. C., \& Zald, D. H. (2012). Effort-based decision-making in major depressive disorder: a translational model of motivational anhedonia. Journal of abnormal psychology, 121(3), 553.

Wabersich, D., \& Vandekerckhove, J. (2014). Extending jags: A tutorial on adding custom distributions to jags (with a diffusion model example). Behavior research methods, 46(1), 15-28.

Wagner, B., Clos, M., Sommer, T., \& Peters, J. (2020). Dopaminergic modulation of human intertemporal choice: A diffusion model analysis using the d2-receptor antagonist haloperidol. Journal of Neuroscience, 40(41), 7936-7948.

Webber, H. E., Lopez-Gamundi, P., Stamatovich, S. N., de Wit, H., \& Wardle, M. C. (2020). Using pharmacological manipulations to study the role of dopamine in human reward functioning: A review of studies in healthy adults. Neuroscience $\mathcal{E}$ Biobehavioral Reviews.

Westbrook, A., van den Bosch, R., Määttä, J., Hofmans, L., Papadopetraki, D., Cools, R., \& Frank, M. J. (2020). Dopamine promotes cognitive effort by biasing the benefits versus costs of cognitive work. Science, 367(6484), 1362-1366.

Wiehler, A., \& Peters, J. (2015). Reward-based decision making in pathological gambling: the roles of risk and delay. Neuroscience Research, 90, 3-14.

Yi, R., Mitchell, S. H., \& Bickel, W. K. (2010). Delay discounting and substance abuse-dependence. Impulsivity: The behavioral and neurological science of discounting, 191-211. 